\title{
The CaPTHUS Scoring Model revisited: Applicability from a UK Cohort with Primary Hyperparathyroidism
}

\author{
${ }^{1}$ Tobias W James, ${ }^{2}$ Michael J Stechman, ${ }^{3}$ David M Scott-Coombes
}

\section{ABSTRACT}

Introduction: Focused parathyroidectomy for primary hyperparathyroidism (pHPT) in patients with a single positive localizing scan may have an unacceptably high recurrence rate unless intraoperative parathyroid hormone (ioPTH) is used. The CaPTHUS score was previously developed to predict singlegland disease in such instances. We evaluated the accuracy of this model in a cohort of patients with pHPT in the UK.

Materials and methods: CaPTHUS scores were calculated from prospectively collected data on consecutive patients undergoing surgery for pHPT [(1 point each for: Preoperative calcium $\geq 3 \mathrm{mmol} / \mathrm{L}$; PTH $\geq 2$ times upper limit; ultrasound (1 point) and sestamibi (1 point) positive for single enlarged gland; concordant positive scans]. Diagnosis of single or multigland disease was confirmed on pathology.

Results: From June 2007 to October 2011, 324 patients (251 female, median age 66, 10-89) underwent surgery for pHPT guided with ioPTH. Single-gland pathology was observed in 291 (89.8\%) patients and multi-gland disease seen in $33(10.2 \%)$. In single-gland disease patients, significantly higher preoperative calcium $(p=0.030)$ and PTH levels $(p=0.033)$ were seen with sensitivities of $65.6 \%$ for ultrasound and $66.0 \%$ for sestamibi scanning. A CaPTHUS score $\geq 3$ was seen in $51.2 \%$ of all patients with a positive predictive value (PPV) for single-gland disease of $99.4 \%$.

Conclusion: A CaPTHUS score $\geq 3$ was accurate at predicting single-gland disease in $>50 \%$ of patients with $\mathrm{pHPT}$, providing a similar PPV and reducing the need for ioPTH implementation in this population. However, recent conflicting literature suggests the CaPTHUS score may not be universally applicable, local audit is recommended before implementation.

Keywords: CaPTHUS, Endocrine surgery, Focused parathyroidectomy, Intraoperative parathyroid hormone, Minimally invasive parathyroidectomy, Primary hyperparathyroidism.

How to cite this article: James TW, Stechman MJ, ScottCoombes DM. The CaPTHUS Scoring Model revisited: Applicability from a UK Cohort with Primary Hyperparathyroidism. World J Endoc Surg 2017;9(1):7-12.

\footnotetext{
${ }^{1}$ Foundation Doctor, ${ }^{2,3}$ Consultant Endocrine Surgeon

${ }^{1}$ Department of Surgery, University Hospital of Wales, Cardiff CF14 4XW, United Kingdom

${ }^{2,3}$ Department of Endocrine and General Surgery, University Hospital of Wales, Cardiff, CF14 4XW, United Kingdom
}

Corresponding Author: Michael J Stechman, Consultant Endocrine Surgeon, Department of Endocrine and General Surgery, University Hospital of Wales, Cardiff, CF14 4XW United Kingdom, Phone: +00442920743349, e-mail: Michael. Stechman@Wales.nhs.uk
Source of support: Nil

Conflict of interest: None

\section{INTRODUCTION}

Primary hyperparathyroidism ( $\mathrm{pHPT}$ ) is a common endocrine disease affecting approximately 22 in 100,000 new patients a year. ${ }^{1}$ Eighty to $90 \%$ of pHPT cases will be due to a single parathyroid adenoma, around $10 \%$ by asymmetric four-gland hyperplasia, and up to $5 \%$ by a double adenoma. Less than $1 \%$ of cases are caused by parathyroid carcinoma. ${ }^{2}$ Parathyroidectomy alleviates the symptoms of hypercalcemia, improves "health-related quality of life" in the majority ${ }^{3}$ and is expected to be curative in more than $95 \%$ of patients. Although bilateral neck exploration (BNE) is still considered the gold standard treatment by many experts, the focused approach by means of a minimally invasive parathyroidectomy (MIP) has shown considerable benefits. These include lower risk of postoperative hypocalcemia, reduced postoperative pain, reduced operation time, and better patient satisfaction. ${ }^{4-6}$ To enable improved selection of patients for MIP, preoperative localization with ultrasonography and scintigraphy (technetium-99m sestamibi scan) has been widely employed. However, in the not uncommon instance of discordance between these scans, or where only one scan is positive and intraoperative parathyroid hormone (ioPTH) measurement is not available, many surgeons are still reluctant to use MIP opting for a conventional BNE instead. ${ }^{4,7}$

One comparative retrospective study found that MIP had a success rate of $99.4 \%$ compared with $97.1 \%$ for BNE. ${ }^{4}$ A second center ${ }^{8}$ reported this focused approach to be curative in $98 \%$ of cases, but unlike the former study, did not use ioPTH testing. Although impressive, both of these large cohort series used careful patient selection to achieve these success rates with the Suliburk et al. ${ }^{8}$ study only performing the focused procedure on patients with concordant neck ultrasound and sestamibi scans.

Objectively, the cost and the marginal benefit in terms of long-term results offered by ioPTH measurement $t^{5,8}$ have meant that it has not been widely adopted in the UK. Importantly, assertions from these and similar reports are also as a result of operations on strictly selected patients with criteria requiring concordant gland localization. 
Interestingly, a study by Westerdahl et $\mathrm{al}^{9}$ suggests that if a minimally invasive procedure is performed with just a single positive localizing study and without ioPTH, the incidence of persistent disease approaches 10\%. In comparison to the former reports, this serves to highlight the beneficial impact, cost savings, and indeed the place of ioPTH measurement in potentially reducing both operative failure and the need for reoperation in this population. Therefore, for centers to offer patientsfocused surgery following a single positive localizing scan, either access to ioPTH or some other method of better determining the presence and location of singlegland disease is required. In 2006, a surgical unit in San Francisco devised the CaPTHUS scoring model as a method of predicting single-gland disease preoperatively in the hope of improving patient selection for focused parathyroidectomy. ${ }^{10}$

\section{OBJECTIVE}

The aim of this study was to determine whether the CaPTHUS scoring model is an accurate method of identifying pHPT due to single-gland disease.

\section{MATERIALS AND METHODS}

\section{Patients and Study Design}

A cohort of consecutive patients undergoing parathyroidectomy for nonfamilial pHPT between June 2007 and October 2011 in a single center were retrospectively studied. Clinical, biochemical, and pathological information on each patient were obtained from a prospectively collected database. Data on body habitus and multinodular goiter were not collected.

Patients were only excluded from the study following negative surgical exploration (due to diagnostic uncertainty), if they were undergoing re-exploration, they had hereditary $\mathrm{pHPT}$ or if they had a carcinoma. The remaining cohort of patients with $\mathrm{pHPT}$ was divided into single and multi-gland disease groups. Single and multiple-gland disease were defined quantitatively by ioPTH measurement following individual gland excision. In multi-gland disease patients, individual gland excision was guided at first qualitatively by appearance, size, and color and then quantitatively by ioPTH measurement during parathyroidectomy. A CaPTHUS score was calculated for each patient, scoring one point each for: Preoperative adjusted calcium $\geq 3 \mathrm{mmol} / \mathrm{L}$; PTH $\geq 2$ times upper limit ( $\geq 14.4 \mathrm{pmol} / \mathrm{L})$; ultrasound scan (USS) positive for a single enlarged gland; sestamibi nuclear medicine scan positive for a single enlarged gland and lastly, concordant scan results positive for a single enlarged gland on the same side of the neck.

\section{Localization Scans and Surgical Approach}

All localizing scans were performed by a single consultant radiologist with more than 10 years of experience with these techniques. Two-dimensional planar sestamibi imaging and USS were performed at the same visit as standard; however, if USS was negative first, single-photon emission computed tomography was used. No other imaging modalities, such as four-dimensional computed tomography or magnetic resonance imaging, were routinely used before primary surgery. If either scan was positive or concordant, patients received ioPTH-guided focused parathyroidectomy. If both scans were negative, a BNE was performed with ioPTH. Choice of unilateral neck exploration (UNE)or MIP was depending on surgeon's preference. The accuracies of the ultrasound and sestamibi scans were determined by comparing radiological findings with the findings at operation and pathological examination. A true positive result was defined as when the localizing scan correctly identified and located a single enlarged parathyroid gland to one side of the neck, not quadrant. A false positive was defined as when the scan incorrectly identified one or more nonenlarged parathyroid glands. A false negative was defined as when the scan failed to identify one or all of the enlarged parathyroid glands (i.e., when the scan failed to correctly identify the patient as having either single or multi-gland disease). True negative results could not occur because all patients had a biochemical diagnosis of pHPT. Concordant scan results were defined as both localizing scans identifying a single enlarged gland on the same side of the neck. ${ }^{10}$ The sensitivity and positive predictive value (PPV) of each imaging modality were also calculated.

\section{Statistical Methods}

Significant statistical difference was defined as $\mathrm{P}<0.05$. Nonparametric data were compared using the MannWhitney U test and parametric data by the Student's t-test. Categorical data were analyzed by the chi-square test with two degrees of freedom. CaPTHUS scores were analyzed to give their sensitivity, specificity, PPV, S and negative predictive value for detecting single-gland disease. To measure the accuracy of the scoring model in our cohort, the area under the receiver operating characteristic (ROC) curve was calculated and measured using Statistical Package for the Social Sciences (version 20.0.0 - IBM).

\section{RESULTS}

During the study period, 335 consecutive patients underwent parathyroidectomy for nonfamilial sporadic pHPT. Eleven patients were excluded: Ten (eight with 
persistent disease and two with recurrent disease) because of uncertainty about the presence of either multiple- or single-gland disease, and one patient with parathyroid cancer. If hypercalcemia returned within 6 months or after 6 months of operation, patients were defined as having either persistent or recurrent disease respectively.

Consequently, 324 patients with pHPT histologically confirmed following parathyroid excision were studied. Patient data are summarized in Table 1. If a drop of 50\% was observed in a patient's ioPTH and they were found to be normocalcemic at 3 months, surgery was defined as curative; by these criteria all 324 patients were defined as cured (overall normocalcemia rate 324/335, 96.7\%). Singlegland disease was found in 291 patients $(89.8 \%)$ and multi-gland disease in 33 (10.2\%). The multi-gland disease group was composed of multiple-gland hyperplasia (23 patients, $7.1 \%$ ) and double adenoma $(10,3.1 \%)$ and is in keeping with the literature. Around 221 patients (68.2\%) underwent focused surgery (unilateral neck exploration or MIP); 101 (31.2\%) patients had BNE, and $2(0.6 \%)$ patients had parathyroidectomy via median sternotomy.

Of 8 patients excluded due to persistent disease, initial imaging with USS was negative in 7 and sestamibi was negative in 6 . Although 3 of these 8 patients did have parathyroid tissue excised at operation, it was diagnosed as normal on histological examination; the other 5 patients had negative explorations. In 4 of these 8 cases, the ioPTH failed to drop below $50 \%$ of baseline, 2 did not have ioPTH recorded and 2 had false positive results where ioPTH did drop below $50 \%$, but later went on to develop persistent disease. Both patients with recurrent disease initially had negative scans. The first had a single-gland excised accompanied by a false positive ioPTH but went on to develop recurrent disease at 12 months. The second patient had a single-gland excised that was described as hyperplastic on histology and developed recurrence at 12 months. All 10 patients had CaPTHUS scores of 0,1 or 2 and so would be predicted to have a higher likelihood of multiple-gland disease.

Although the differences between the ages of the single and multi-gland groups were not significant, there was a trend toward patients with multi-gland disease being older (Mann-Whitney U: $\mathrm{p}=0.070$ ). There was no significant difference in gender proportions between single and multi-gland disease groups, nor a significant difference between the groups' gland weights (MannWhitney U: $p=0.173$; Table 1).

When preoperative serum biochemistry was analyzed, multi-gland disease patients had a significantly lower adjusted serum calcium concentration (Student's t-test: $p=0.030$ ) and significantly lower serum PTH concentrations (Mann-Whitney U: $\mathrm{p}=0.033$ ) as shown in Table 1. Ultrasound and sestamibi scanning were more likely to be positive and correct in single-gland disease (MannWhitney U: $\mathrm{p} \leq 0.001$ ). The ultrasound and sestamibi scans had a sensitivity of correctly predicting single-gland disease of $65.6 \%$ and $66.0 \%$ respectively and a PPV of $95.9 \%$ and $97.4 \%$ respectively. The PPV of two concordant scans for single-gland disease was $96.15 \%$ and this was observed in 156 patients (48.1\%).

When CaPTHUS scores were individually calculated for each patient, a score of 3 or higher was found in 166 $(51.2 \%)$ and the sensitivity and PPV for single-gland disease were $56.7 \%$ and $99.4 \%$ respectively (Table 2 ). The area under the receiver-operator characteristic curve for the CaPTHUS scoring model in our cohort was 0.806 suggesting that the scoring criteria performed well at classifying patients into single- and multiple-gland disease groups (Graph 1).

Table 1: Characteristics of pHPT patients and comparison between single and multiple-gland pHPT groups

\begin{tabular}{|c|c|c|c|c|c|}
\hline \multicolumn{2}{|l|}{ Clinical characteristics } & Overall values & Single-gland & Multi-gland & $p$-value \\
\hline \multicolumn{2}{|l|}{ No. of patients $(n)$} & 324 & $291(89.8 \%)$ & $33(10.2 \%)$ & \\
\hline \multicolumn{2}{|l|}{ Male:Female } & $73: 251$ & $68: 223$ & $5: 28$ & \\
\hline \multicolumn{2}{|l|}{ Median age, years } & $66(10-89)$ & $65(10-89)$ & $71(14-84)$ & $0.070^{*}$ \\
\hline \multicolumn{2}{|l|}{ Mean serum calcium $\pm \mathrm{SD}, \mathrm{mmol} / \mathrm{L}$} & $2.86 \pm 0.22$ & $2.87 \pm 0.23$ & $2.79 \pm 0.17$ & $0.030 \#$ \\
\hline \multicolumn{2}{|l|}{ Median serum PTH, pmol/L } & $13.4(2.84-139)$ & $14.4(2.84-139)$ & $11.2(3.50-42.5)$ & $0.033^{*}$ \\
\hline \multicolumn{2}{|l|}{ Median gland weight, milligrams } & $461(50-9000)$ & $600(50-9000)$ & $456(150-2012)$ & $0.173^{*}$ \\
\hline \multicolumn{2}{|c|}{ Sensitivity of localisation scans, (\%) Ultrasound } & 60.6 & 65.6 & 4.17 & $<0.001^{*}$ \\
\hline \multirow{3}{*}{ Positive predictive value, (\%) } & Sestamibi & 60.6 & 66.0 & 3.70 & $<0.001^{*}$ \\
\hline & Ultrasound & 91.6 & 95.9 & 10.0 & \\
\hline & Sestamibi & 94.0 & 97.4 & 14.3 & \\
\hline \multirow{4}{*}{$\begin{array}{l}\text { Surgical approach, patient } \\
\text { numbers, }(\%)\end{array}$} & Unilateral surgical exploration & $201(62.0)$ & $200(68.7)$ & $1(3.0)$ & \\
\hline & Bilateral surgical exploration & $99(30.6)$ & $67(23.0)$ & $32(97.0)$ & \\
\hline & Minimally invasive parathyroidectomy & $22(6.8)$ & $22(7.6)$ & $0(0)$ & \\
\hline & Median sternotomy & $2(0.6)$ & $2(0.7)$ & $0(0)$ & \\
\hline
\end{tabular}

SD: Standard deviation; *Mann-Whitney U test; Student's t-test 


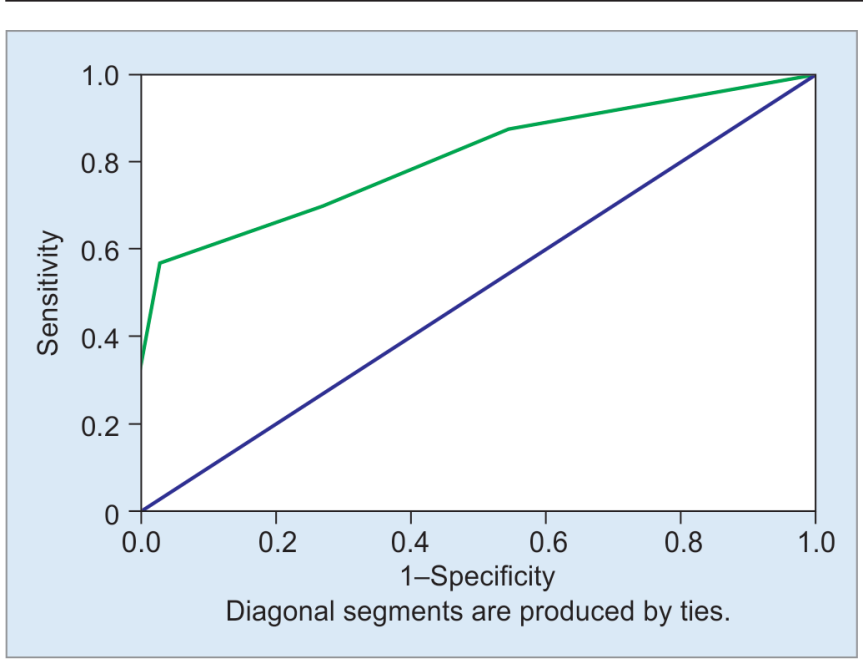

Graph 1: Receiver operator characteristic curve for the CaPTHUS scoring model in a UK cohort of patients

False negative localization results were identified in approximately one-third of all scans performed. Sestamibi and USS failed to detect a single enlarged gland in $33.3 \%$ of cases. In those with multiple-gland disease, sestamibi and USS identified a single enlarged gland in $6(15.2 \%)$ and $9(27.3 \%)$ patients respectively (Table 3$)$. An anomalous CaPTHUS score of $\geq 3$ was seen in only one patient with multiple-gland disease (Table 4). This patient underwent a BNE due to a known concurrent $2 \mathrm{~cm}$ thyroid nodule, potentially accounting for the false positive scans giving us little concern over the integrity of the scoring model.

Intraoperative PTH measurement was used in 298 (92.0\%) operations and successfully accounted for all diseased glands in 296 (99.3\%) cases where it was used. The remaining two patients had false negative ioPTH results, where ioPTH did not drop below $50 \%$ of baseline, and so underwent BNE. Both were cured postoperatively and remained normocalcemic at 12 months with histologically confirmed single adenoma. Thirteen patients (4.4\%) benefited from ioPTH where it successfully recognized previously unidentified multi-gland disease preventing recurrence or persistent disease. All of these patients had a CaPTHUS score of 0,1 or 2 suggesting a score of 2 or less could be a criterion for ioPTH.

Of the patients with proven single-gland disease and a score of $\geq 3,19(6 \%)$ of 170 underwent BNE and the remaining 131 had focused surgery (UNE or MIP). These 19 patients could have been offered focused surgery had the CaPTHUS score been implemented at negligible cost.

\section{DISCUSSION}

In this consecutive cohort of patients with pHPT, the CaPTHUS scoring model reliably identified single-gland disease in 51.2\% (PPV: 99.4\%) of affected patients when a score of 3 was set as the minimum (area under the ROC curve of 0.806). A score of 3 or higher is crucial for its applicability in clinical practice. For example, in a patient with an adjusted serum calcium concentration $\geq 3 \mathrm{mmol} / \mathrm{L}$ and a serum PTH concentration $\geq 2$ times the upper limit of normal, with just one localizing scan positive for an enlarged single- gland (USS or sestamibi), the surgeon can be more than $99 \%$ confident that the cause

Table 2: CaPTHUS score analysis

\begin{tabular}{llllllll}
\hline \multirow{2}{*}{ CaPTHUS score } & No. of patients (\%) & Sens. & Spec. & PPV & NPV & $\begin{array}{l}\text { Mean serum calcium, } \\
\text { mmol/L } \pm S D\end{array}$ & Median PTH, pmol/L \\
\hline 0 & $52(16.0)$ & 12.7 & 54.6 & 71.2 & 6.6 & $2.77 \pm 0.09$ & $10.90(3.1-13.9)$ \\
$\geq 1$ & $272(84.0)$ & 87.3 & 45.5 & 93.4 & 28.9 & $2.88 \pm 0.24$ & $16.00(2.84-139)$ \\
$\geq 2$ & $213(65.7)$ & 69.8 & 69.7 & 95.3 & 20.7 & $2.91 \pm 0.26$ & $17.70(2.84-139)$ \\
$\geq 3$ & $166(52.5)$ & 56.7 & 97.0 & 99.4 & 20.3 & $2.91 \pm 0.27$ & $16.60(2.84-125)$ \\
$\geq 4$ & $90(27.8)$ & 31.0 & 100 & 100 & 14.1 & $2.97 \pm 0.30$ & $23.20(9.60-125)$ \\
5 & $26(8.0)$ & 9.0 & 100 & 100 & 11.1 & $3.31 \pm 0.34$ & $32.90(15.0-125)$ \\
\hline
\end{tabular}

Sens: Sensitivity = Proportion of the single-gland primary hyperparathyroidism (pHPT) patients picked up by the score; Spec: Specificity = Proportion of patients without single-gland pHPT not picked up correctly by the score; PPV: Positive predictive value = The proportion of patients with true positive scores for single-gland pHPT; NPV: Negative predictive value $=$ The proportion of patients not getting the score that also did not have single-gland disease; SD: Standard deviation

Table 3: Comparison of predictive factors between single and multi-gland primary hyperparathyroidism groups

\begin{tabular}{lllr}
\hline Predictive factor & $\begin{array}{l}\text { Single-gland } \\
\text { disease (\%) }\end{array}$ & $\begin{array}{l}\text { Multi-gland } \\
\text { disease (\%) }\end{array}$ & $p$-value \\
\hline Patient numbers & $291(89.8)$ & $33(10.2)$ & $0.404^{\#}$ \\
Total serum calcium level $\geq 3.00 \mathrm{mmol} / \mathrm{L}$ & $49(16.8)$ & $2(6.1)$ & $0.156^{\#}$ \\
PTH level $\geq 2$ times upper limit of normal & $144(49.5)$ & $11(33.3)$ & $<0.001^{\#}$ \\
Neck USS positive for 1 enlarged parathyroid gland & $196(67.4)$ & $9(27.3)$ & $<(15.2)$ \\
Sestamibi scan positive for 1 enlarged parathyroid gland & $196(67.4)$ & $1(3.0)$ & $<0.001^{\#}$ \\
Concordant USS + sestamibi results for 1 enlarged gland on same side & $155(53.3)$ & 1 \\
\hline
\end{tabular}

\section{\#Chi-square test with 2 degrees of freedom}


Table 4: Comparing the number of patients with each CaPTHUS score in the single and multi-gland disease patient groups

\begin{tabular}{lll}
\hline $\begin{array}{l}\text { CaPTHUS } \\
\text { score }\end{array}$ & Single-gland disease & $\begin{array}{l}\text { Multiple-gland } \\
\text { disease }\end{array}$ \\
\hline 0 & 37 & 15 \\
1 & 51 & 8 \\
2 & 38 & 9 \\
3 & 75 & 1 \\
4 & 64 & 0 \\
5 & 26 & 0 \\
\hline
\end{tabular}

is single-gland disease, and that a MIP or unilateral exploration can be performed without need for ioPTH.

The costs and benefits of ioPTH measurement have been keenly debated in the literature, so any data that can add important features to the discussion should be welcomed. ${ }^{5,11-13}$ The earlier mentioned study by Suliburk et $\mathrm{al}^{8}$ demonstrated that using concordant imaging essentially a CaPTHUS score of $\geq 3$ - as the criteria to perform MIPs without ioPTH could achieve a success rate of $98 \%$. Similarly, Goldstein et $\mathrm{al}^{11}$ found that MIP procedures without routine ioPTH still provided an "excellent cure rate" for $\mathrm{pHPT}$, but their criteria for performing an MIP required a positive sestamibi scan. Evaluation of these studies with reference to the data presented, suggests that neither a positive sestamibi scan nor concordant scans, have to be necessary criteria for a successful MIP when the additive value from biochemical results are incorporated. In some instances, the benefits of the focused procedure without ioPTH can be achieved with the same confidence when only the USS is positive along with calcium $\geq 3 \mathrm{mmol} / \mathrm{L}$ and $\mathrm{PTH} \geq 2$ times the upper limit of normal.

A study produced by Stalberg et a ${ }^{14}$ helps to reinforce this point. They found that the use of ioPTH does not add any significant value to decision making during MIP but that the high cure rate is built around appropriate selection of patients based on localizing studies. In contrast, Barczynski et al ${ }^{15}$ suggested that ioPTH provides "substantial" value to surgeons performing focused parathyroidectomy, particularly when only one scan is positive. This is where a CaPTHUS score $\geq 3$ can be useful, giving surgeons greater confidence that the patient has single-gland pHPT.

Although our summary broadly fell in line with that of Kebebew et $\mathrm{al}^{10}{ }^{10}$ we did not find the " $100 \% \mathrm{PPV}^{\text {" or }}$ $100 \%$ specificity for a CaPTHUS score of $\geq 3$. However, a score of 3 or above did apply to $51.2 \%$ of our pHPT patients compared with 35\% in Kebebew's study. One reason for this finding might be that patients selected for surgery in the North American cohort may have had smaller tumors that are less likely to be apparent on localization scans, perhaps as a result of being selected for surgery at an earlier stage in the disease course. It has been demonstrated previously that gland weight correlates positively with successful localization ${ }^{16}$ and resultantly, these tumors are harder to localize. As gland weight data is not provided in that study, this assertion cannot be verified. ${ }^{10}$ Compared with a recent review on localization techniques employed in PHPT management, ${ }^{17}$ the sensitivities and specificities of both the ultrasound and sestamibi studies in our report appear relatively low. It is possible and well-documented that body habitus and examiner experience could account for reduced success with these techniques, especially with USS. However, with all imaging performed by the same experienced senior radiology consultant, this aspect seems less likely. Although we do not have specific data on the relative body habitus of this cohort, one can reasonably assume correlation with local population data from the 2010 Welsh Health Survey ${ }^{18}$ identifying $57 \%$ of adults as overweight (BMI 25-30) including $22 \%$ as obese (BMI >30). This could contribute to the lower than previously reported sensitivities of these imaging modalities, particularly USS. Therefore, to have seen a larger percentage of patients with CaPTHUS scores $\geq 3$ in our cohort might imply that raised biochemical findings contributed to their scores in more instances. This could in turn account for larger adenomas and more severe disease that may reflect further differences in local disease presentation compared with Kebebew et al ${ }^{10}$.

Recently, another North American centre ${ }^{19}$ published data similarly assessing the CaPTHUS score in their population of PHPT patients. They conversely concluded that had the CaPTHUS score been used to dictate operative approach and omission of ioPTH in the $22.5 \%$ of patients with a score $\geq 3$ (307 out of 1,421 patients), around 10\% of multi-gland patients would have had their operations prematurely terminated. They infer eliminating ioPTH should not be a routine. In contrast to the presented study where single adenoma was confirmed in $89.8 \%$ of cases, it was seen in $78 \%$ of their patients. Only about a third of the cohort was dual scanned resulting in fewer scores of $\geq 3$ ( $22.5 \%$ vs $51.2 \%$ in our cohort). Of the patients that were dual scanned, $48 \%$ had a score $\geq 3$ but the PPV of identifying single-gland disease remained insignificantly different to their original population at $91 \%$. Elfenbein et $\mathrm{a}^{19}$ speculate that the discrepancies seen may be due to "milder forms" of the disease and that not all patients had dual scans. Following this, their larger percentage of multiple-gland disease might reflect their larger amounts of milder disease presentation. They surmise that using a score of $\geq 4$ as the cut off would provide greater accuracy but worse applicability in their cohort. Interestingly, like our study, the results from Wisconsin are at variance with 
the original model. This conflicting advice implies that although the CaPTHUS score appears accurate and useful in some instances, it might have significant variability across populations suggesting general inference from studies may be more limited than hoped. This could have many possible contributing causes including differences in reporting patterns (early vs late), climate, diet, and genetics.

\section{CONCLUSION}

A CaPTHUS score of $\geq 3$ was accurate at predicting singlegland pHPT in more than half of the patients studied and could be useful in providing the surgeon with confidence to use a minimally invasive approach, even when ioPTH is not available. However, as the CaPTHUS score appears to work differently across other populations, the potential benefits from its application are not universal. We recommend that local centers should evaluate the model using a quick audit before implementation.

\section{REFERENCES}

1. Adami S, Marcocci C, Gatti D. Epidemiology of primary hyperparathyroidism in Europe. J Bone Miner Res 2002 Nov;17 (Suppl 2):N18-23.

2. Kebebew E, Clark OH. Parathyroid adenoma, hyperplasia, and carcinoma: localization, technical details of primary neck exploration, and treatment of hypercalcemic crisis. Surg Oncol Clin N Am 1998 Oct;7(4):721-748.

3. Mack LA, Pasieka JL, Asymptomatic primary hyperparathyroidism: a surgical perspective. Surg Clin North Am 2004 Jun;84(3):803-816.

4. Udelsman R, Lin Z, Donovan P. The superiority of minimally invasive parathyroidectomy based on 1650 consecutive patients with primary hyperparathyroidism. Ann Surg 2011 Mar;253(3);585-591.

5. Wong IY, Lang BH. Minimally invasive parathyroidectomy for primary hyperparathyroidism - current views, issues and controversies, hyperparathyroidism. In: Soto GD, PuigDomingo M, editors- Chapter 3. 2012. p. 21-34.

6. Valdemarsson S, Lindergård B, Tibblin S, Bergenfelz A. Increased biochemical markers of bone formation and resorption in primary hyperparathyroidism with special reference to patients with mild disease. J Intern Med 1998 Feb;243(2):115-122.

7. Spiros D, Nikolaos R, Ioannis C. Minimally invasive parathyroidectomy in patients with previous endocrine surgery. JSLS 2011 Oct-Dec;15(4):499-503.
8. Suliburk J, Sywak M, Sidhu S, Delbridge LW. 1000 minimally invasive parathyroidectomies without intra-operative parathyroid hormone measurement: lessons learned. ANZ J Surg 2011 May;81(5):362-365.

9. Westerdahl J, Bergenfelz A. Sestamibi scan-directed parathyroid surgery: potentially high failure rate without measurement of intraoperative parathyroid hormone. World J Surg 2004 Nov;28(11):1132-1138.

10. Kebebew E, Hwang J, Reiff E, Duh QY, Clark OH. Predictors of single-gland vs multigland parathyroid disease in primary hyperparathyroidism: a simple and accurate scoring model. Arch Surg 2006 Aug;141(8):777-782.

11. Goldstein R, Billheimer D, Martin WH, Richards K. Sestamibi scanning and minimally invasive radioguided parathyroidectomy without intraoperative parathyroid hormone measurement. Ann Surg 2003 May;237(5):722-731.

12. Yen TW, Wilson SD, Krzywda EA, Sugg SL. The role of parathyroid hormone measurements after surgery for primary hyperparathyroidism. Surgery 2006 Oct;140(4);665-674.

13. Palazzo FF, Delbridge LW. Minimal-access/minimally invasive parathyroidectomy for primary hyperparathyroidism. Surg Clin North Am 2004 Jun;84(3):717-734.

14. Stalberg P, Sighu S, Sywak M, Robinson B, Wilkinson M, Delbridge L. Intraoperative parathyroid hormone measurement during minimally invasive parathyroidectomy: does it "value-add" to decision-making? J Am Coll Surg 2006 Jul;203(1):1-6.

15. BarczynskiM,KonturekA,CichonS,Hubalewska-DydejczykA, Golkowski F, Huszno B. Intraoperative parathyroid hormone assay improves outcomes of minimally invasive parathyroidectomy mainly in patients with a presumed solitary parathyroid adenoma and missing concordance of preoperative imaging. Clin Endocrinol (Oxf) 2007 Jun;66(6): 878-885.

16. Calva-Cerqueira D, Smith BJ, Hostetler ML, Lal G, Menda Y, O'Dorisio TM, Howe JR. Minimally invasive parathyroidectomy and preoperative MIBI scans: correlation of gland weight and preoperative PTH. J Am Coll Surg 2007 Oct;205 (Suppl 4):S38-44.

17. Kunstman JW, Kirsh JD, Mahajan A, Udelsman R. Cinical review: Parathyroid Localization and Implications for Clinical Management. J Clin Endocrinol Metab 2013 Mar;98(3): 902-912.

18. Griffiths E, Roberts C The Welsh Health Survey 2010. [Internet] [cited 2014 March]. Available from: www.wales.gov.uk/ statistics-and-research/welsh-health-survey.

19. Elfenbein DM, Weber S, Schneider DF, Sippel RS, Chen H. CaPTHUS scoring model in primary hyperparthyroidism: can it eliminate the need for ioPTH Testing? Ann Surg Oncol 2015 Apr;22(4):1191-1195. 